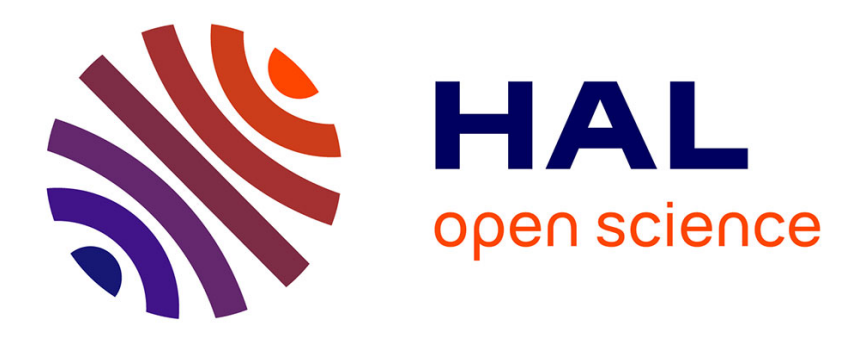

\title{
From modules to lattices: Insight into the genesis of Dedekind's Dualgruppen
}

\author{
Emmylou Haffner
}

\section{To cite this version:}

Emmylou Haffner. From modules to lattices: Insight into the genesis of Dedekind's Dualgruppen. British Journal for the History of Mathematics, 2018, 10.1080/17498430.2018.1555928 . hal02426462

\section{HAL Id: hal-02426462 \\ https://hal.science/hal-02426462}

Submitted on 6 Jan 2020

HAL is a multi-disciplinary open access archive for the deposit and dissemination of scientific research documents, whether they are published or not. The documents may come from teaching and research institutions in France or abroad, or from public or private research centers.
L'archive ouverte pluridisciplinaire HAL, est destinée au dépôt et à la diffusion de documents scientifiques de niveau recherche, publiés ou non, émanant des établissements d'enseignement et de recherche français ou étrangers, des laboratoires publics ou privés. 


\title{
From modules to lattices, insight into the genesis of Dedekind's Dualgruppen
}

\author{
Emmylou Haffner \\ Bergische Universität Wuppertal \& SPHERE \\ Preprint, please do not cite this version
}

\begin{abstract}
When Dedekind introduced the notion of module, he also defined their divisibility and related arithmetical notions (e.g. LCM of modules). The introduction of notations for these notions allowed Dedekind to state new theorems, now recognized as the modular laws in lattice theory. Observing the dualism displayed by the theorems, Dedekind pursued his investigations on the matter. This led him, twenty years later, to introduce Dualgruppen, equivalent to lattices [Dedekind, 1897, Dedekind, 1900]. After a brief exposition of the basic elements of Dualgruppe theory, and with the help of his Nachlass, I show how Dedekind gradually built his theory through layers of computations, often repeated in slight variations and attempted generalizations. I study the tools he devised to help and accompany him in his computations. I highlight the crucial conceptual move that consisted in going from investigating operations between modules, to groups of modules closed under these operations. By using Dedekind's drafts, I aim to highlight the concealed yet essential practices anterior to the published text.
\end{abstract}

Keywords: history of mathematics; Richard Dedekind; mathematical drafts; lattice theory.

\section{Introduction}

In his celebrated [Dedekind, 1871], Richard Dedekind introduced the notion of module, a "system $\mathfrak{a}$ of real or complex numbers $\alpha$ whose sums and differences themselves belong to $\mathfrak{a}$ " [Dedekind, 1871, 42]. Modules play a central role in Dedekind's number theory. To develop module theory, Dedekind defined a divisibility relation for modules: a module $\mathfrak{a}$ is divisible by a module $\mathfrak{b}$ if $\mathfrak{a} \subset \mathfrak{b}$. This divisibility relation sets the ground for related arithmetical notions and for the arithmetical methodology that prevailed in many of his mathematical works. Of particular interest, for this paper, are the notions of least common multiple and of greatest common divisor of modules: the LCM of $\mathfrak{a}$ and $\mathfrak{b}$ is $\mathfrak{a} \cap \mathfrak{b}$, and the 
GCD of $\mathfrak{a}$ and $\mathfrak{b}$ is the module composed by all the numbers $\alpha+\beta$ with $\alpha$ and $\beta$ respectively running through all numbers of $\mathfrak{a}$ and $\mathfrak{b}$.

In [Dedekind, 1877a], Dedekind introduced notations for divisibility, LCMs and GCDs of modules: $\mathfrak{a}$ divides $\mathfrak{b}$ is denoted by $\mathfrak{a}<\mathfrak{b}$ or $\mathfrak{a}>\mathfrak{b} ;$ the GCD of $\mathfrak{a}$ and $\mathfrak{b}$ by $\mathfrak{a}+\mathfrak{b}$; and their LCM by $\mathfrak{a}-\mathfrak{b}$. This allowed him to state new theorems such as, for three modules $\mathfrak{a}, \mathfrak{b}, \mathfrak{c}$ with $\mathfrak{a}<\mathfrak{b}$ :

$$
\begin{aligned}
& (\mathfrak{a}+\mathfrak{b})-(\mathfrak{a}+\mathfrak{c})=\mathfrak{a}+(\mathfrak{b}-(\mathfrak{a}+\mathfrak{c})) \\
& (\mathfrak{a}-\mathfrak{b})+(\mathfrak{a}-\mathfrak{c})=\mathfrak{a}-(\mathfrak{b}+(\mathfrak{a}-\mathfrak{c})) .
\end{aligned}
$$

These theorems are equivalent to

$$
(\mathfrak{a}+\mathfrak{b})-(\mathfrak{a}+\mathfrak{c})=(\mathfrak{a}-\mathfrak{b})+(\mathfrak{a}-\mathfrak{c})
$$

Or again

$$
\mathfrak{a}+(\mathfrak{c}-\mathfrak{b})=(\mathfrak{a}+\mathfrak{c})-\mathfrak{b} .
$$

The last equality corresponds to what is now called the 'modular law' in lattice theory.

Dedekind noted that these "characteristic theorems" for modules display a "dualism that holds throughout for the notions of GCD and LCM" [Dedekind, 1877b, 121], that is, any true formula expressed in terms of + and - can be transformed into another true formula by switching these symbols. Interested by this dualism, Dedekind pursued that road, and eventually introduced the notion of Dualgruppe (equivalent to our modern lattice). ${ }^{1}$

The notion first appears in [Dedekind, 1894], under the name Modulgruppe. Dedekind presented his Dualgruppe theory in [Dedekind, 1897] and [Dedekind, 1900]. This was, in his words, obtained "not without great effort" [Dedekind, 1897, 113] and indeed after two decades of work. For Dedekind, these works were introducing a new concept, the Dualgruppe, which could be applied in many domains and thus be fruitful for mathematiciansmuch like ideals, modules or groups, in his opinion. However, these two papers did not attract much attention after their publication, and only became more widely known after 1930 with Birkhoff's work on lattices. ${ }^{2}$

It is well known that Dedekind considered that for a better exposition of a theory, it should avoid, as much as possible, the resort to computational or algorithmic methods. Rather, as he stated at several occasions, ${ }^{3}$ a theory should be based upon concepts, following the principles established by Riemann in function theory. Yet, it is worth mentioning

\footnotetext{
${ }^{1}$ Although a Dualgruppe is formally equivalent to a lattice, the two concepts were developed independently, and are in fact very different in their conception and uses. I will thus use Dedekind's terminology.

${ }^{2}$ In fact, Birkhoff's first works on lattices are independent of Dedekind's Dualgruppen. It was Oysten Öre, who was at the time editing Dedekind's Gesammelte Werke, who brought these works to Birkhoff's attention [Birkhoff, 1934, 200].

${ }^{3}$ See, for example, [Dedekind, 1932, III, 468], or [Dedekind, 1877a, 102].
} 
that analyses of Riemann's Nachlass ${ }^{4}$ showed that his "conceptualism" was, if not overestimated, at least overemphasized and somewhat blind to the preliminary phases of research. ${ }^{5}$ As Edwards puts it, while Riemann may have been "primarily interested in grand general abstract concepts", he "did not [however] venture into these higher realms without doing a lot of serious computation to lay the groundwork for his flights" [Edwards, 2010, 64]. Riemann's 'conceptual mathematics' indeed relies on a great amount and a deep, exceptional mastery of computations, which were a step in the mathematical research, subsequently hidden during the writing for publication. I would like to show how computations played a similar role in the development of Dedekind's concept of Dualgruppe, and to understand and clarify these preliminary researches as observable in his Nachlass. Indeed, while Dedekind's computational abilities might not compare to Riemann's, his manuscripts on module theory $^{6}$ from the late 1870 s to after 1900 contain an impressive amount of computations. They show that Dedekind's research process, here, implied many layers of computations that allowed him to explore and unfold module theory and the properties of its operations. ${ }^{7}$ The computations and the stepwise generalization of the concept, however, largely disappeared from the published exposition of the theory, which is very general and abstract - or even axiomatic, as stated by [Schlimm, 2011].

After a brief exposition of the content of Dedekind's Nachlass and basic elements of Dualgruppe theory, I will show how Dedekind's initial interest for the operations defined between modules for his number-theoretical works, led him to explore the possibilities offered by these operations and by the dualism of the operations, with series of examples, tables, and calculations. By doing so, Dedekind was looking for remarkable and generally valid properties and attempting to identify which properties should be considered as "fundamental" ones. He thus gradually built his theory through many layers of computations, often repeated in slight variations and attempted generalizations, while also devising tools to support or even help the computations. From the computations emerge two main ten-

\footnotetext{
${ }^{4}$ [Edwards, 2010]

${ }^{5}$ The distinction that I am making, here, between the preliminary phases of research and the published work is slightly different from the traditional distinction between the context of discovery and the context of justification. Indeed, the justification of a result in mathematics is taken to be its proof, which certainly can and does happen during the preliminary phases of research. Rather, it is a distinction between the mathematicians' modes of writing for research (which can be multiple) and for publication (which answer specific disciplinary criteria). It can be linked to the idea that "mathematics has a front and a back" [Hersch, 1991].

${ }^{6}$ I will use the term "module theory" as an actor's category, that is, as Dedekind himself designates the set of definitions and results he established for modules (and similarly, for example, for ideal theory or Dualgruppe theory), which he considered as fairly clearly defined and circumscribed within his works, from its first introduction in [Dedekind, 1871]. This should not be taken as meaning that module theory is an established discipline within the mathematical community at the time. As [Corry, 2004] showed, the meaning of many of the concepts of structural algebra, notably in Dedekind's works, were not fixed at the end of the 19th century.

${ }^{7}$ In the over 600 folios of manuscripts on module theory, Modulgruppen and Dualgruppen kept in Göttingen archives, less than 200 pages are fully written (as opposed to mere computations and tables).
} 
dencies in Dedekind's research, which will each be the subject of the two following sections. On one hand, Dedekind shows a deeper interest for the operations inasmuch as they can be applied to other objects than modules, and he tries to study them independently from the nature of the operands. On the other hand, Dedekind's focus slowly shifts from the operations between modules towards what he calls "groups" formed by modules (which are systems closed under the previously defined operations). ${ }^{8}$ These two moves are independent but are eventually merged, which is how the notion of Dualgruppe is introduced by Dedekind.

\section{Material and methods}

This paper relies on the manuscripts and drafts kept in Dedekind's Nachlass. ${ }^{9}$ These drafts were working tools for Dedekind. They reveal the concealed layers of mathematics that happened before the publication. As such, they offer the historian of mathematics a possibility to analyze and clarify the preliminary and intermediary states of mathematical research, the essential yet hidden practices that are often concealed by the mathematician when they format their work for communication to the scientific community. Dedekind himself makes a clear distinction between the published presentation of the theory, and the "historical presentation of the chain of thought" [Edwards et al., 1982, 63]. How one comes to invent a concept and how one choses to define it are often different things. The way in which a mathematician choses to define a concept is historically situated, and shaped by the disciplinary norms of legitimacy and rigour established by the scientific community. Such a definition is chosen as the most accurate presentation, and is ultimately the one that should be remembered. My aim, here, is not to deny this, but to shift the attention to the mathematics 'in the making', to better understand the shaping of mathematical ideas, practices and theories.

It would be unrealistic to attempt a precise chronology of Dedekind's manuscripts

\footnotetext{
${ }^{8}$ The term "group", in Dedekind's writings, is used with a relatively large and fluid meaning. Most of the time, Dedekind used the word "group" as he understood it from Galois's works (which he was the first to teach in Germany [Dedekind, 1858]): a set of elements closed under one or two given operations. The properties of the operations would give defining properties of the "group" in question - properties like associativity were not, for example, included in his general idea of a "group". It is the use made in his drafts on Dualgruppen. In this paper, I will use the term "group" as an actor's category and follow Dedekind's use of the word. Note that outside of Dedekind's works, at the end of the 19th century, the term "group" also covered several different meanings or understandings of the concept (see [Wussing, 1984, Ehrhardt, 2012]). There was, thus, no "group theory" as we understand it today.

Finally, as an anonymous referee pointed out to me, one can also observe, in a draft of [Dedekind, 1888] reproduced in [Dugac, 1976, 296], that Dedekind's chains were first called "groups". It is one of many occurrences, in Dedekind's manuscripts, of changing terminology as part of the research process - see, below, the changing names of Dualgrupen. One can also mention that in a manuscript from before 1871 [Dedekind, 1870], Dedekind used the word "Körper" to denote a system of points with certain topological properties, which had nothing in common with the algebraic notion of field introduced in [Dedekind, 1871].

${ }^{9}$ See the section 2.1 .
} 
and research process, as the manuscripts are usually not dated, and can only be, at best, approximately dated (see below). However, it is possible to distinguish the main movements of the research, whose exposition will constitute the guiding thread of this paper. Using the manuscripts as a way to enter into Dedekind's workshop and to try to understand the way(s) in which he elaborated the concept of Dualgruppe, my analysis will rely on methodological insights drawn from studying the drafts, notebooks and other manuscripts left by scientists, in particular in [Holmes et al., 2003] and [Barberousse and Pinon, 2003]. Besides, as I will be using the manuscripts to understand the text as a process and as a tool for the mathematician's work, my analysis will also rely, from a methodological viewpoint, on works that emphasized the fruitfulness of joining the history of text to the history of science and of mathematics, such as [Chemla, 1995, Chemla, 2004]. ${ }^{10}$

Dedekind's works on lattice theory did not attract as much attention as some of his other works. On the matter, one can see [Mehrtens, 1979], [Corry, 2004, 121-128] and [Schlimm, 2011]. The archival material on which this paper relies has not, as far as I am aware, been studied so far.

\subsection{Notes on the archival material}

In Richard Dedekind's archive, kept at the Niedersächsische Staats- und Universitätsbibliothek in Göttingen, nine files contain documents concerned with the genesis of the Dualgruppe concept : Cod. Ms. R. Dedekind III 14, X 9, X 10, X 11-1, X 11-2, XI 1, XI 2, XI 3-1 and XI 3-2. They contain a total of 568 folios.

- Cod. Ms. R. Dedekind III 14 (1890s) is the only one that can be related to [Dedekind, 1897] and exclusively to it.

It mostly contain documents laying the grounds of the 1897 paper, with a lot of fully written material, in particular next to last and last draftings of [Dedekind, 1897]. Since I am, here, mostly interested in the phases of research before writing a paper for publication, they are slightly out of the scope of my investigation and will not be used. I should add that this file is not devoid of the kind of preliminary researches that I will consider. However Dedekind uses a strange notation, which can be described as figurative numbers: three modules are denoted by the number 1,2 and 3 , and their product by $12,23,13$, etc. ${ }^{11}$ The notation is difficult to understand, especially insofar as Dedekind keeps trying to refine it and apparently introduces several versions of it. For brevity's sake, these manuscripts had to be excluded from the present paper.

\footnotetext{
${ }^{10}$ In addition, a further step in studying the genesis of Dedekind's Dualgruppen will be a critical genetic edition of the manuscripts (see [de Biasi, 2011, Grésillon, 2016]), a work in progress on http: //eman-archives.org/Dedekind/ which will allow for a better and more global grasp of the research and writing process.

${ }^{11} \mathrm{~A}$ version of this notation is used in [Dedekind, 1897].
} 
- Five of the files relate to either the general considerations on Dualgruppen in [Dedekind, 1897] or to [Dedekind, 1900]:

- Cod. Ms. R. Dedekind X 9 (after 1884 up to 1900);

- Cod. Ms. R. Dedekind X 10 (mid-1890s);

- Cod. Ms. R. Dedekind X 11-1 (late 1870s to early 1890s);

- Cod. Ms. R. Dedekind XI-1 (1894 to 1897, and a couple of folios from the late 1870s);

- Cod. Ms. R. Dedekind XI-2 (late 1890s).

It is difficult to pinpoint exactly which file is related to which paper. Indeed, their content consists of researches and computations on modules, groups, the duality and various properties of the operations, Modulgruppen, Dualgruppen, which can be found in both papers. In addition, we have no certainty that Dedekind himself put all the folios together in each file. As explained at the beginning of the archives' catalog, the Nachlass was donated to the Göttingen archive by Dedekind's heirs in 1931 (fifteen years after Dedekind's death). Many of the files appear to be very fragmentary. Despite the seemingly thematic organization of the Nachlass, it can be doubtful that all the folios put together do indeed form a consistent thematic unit on the same researches.

- Cod. Ms. Dedekind X-11-2 contains manuscripts from the 1890s and seems to be the file with the most material related precisely to [Dedekind, 1900].

- Cod. Ms. Dedekind XI 3-1, XI 3-2, both entitled "On the article (1900) : On the Dualgruppe generated by three modules" by the Göttingen archives, contain manuscripts written after the publication of the 1900 paper. They fall out of the scope of the present paper - it should, however, be noted that they do exhibit some similar traits on Dedekind's practice of computations.

The dating of these files is difficult, as Dedekind himself very rarely gives such indications on his drafts. Time intervals can be given using references to other works (mainly Dirichlet's Vorlesungen and Dedekind's own works, occasionally Ernst Schröder's or Georg Frobenius'), or indications given by the sheets on which Dedekind was writing. Indeed, he often wrote on the back of advertisements, invoices, administrative papers, letters, or drafts of his own (published) papers. However, a discrepancy of up to ten years can be observed, for example on manuscripts containing both a reference to a published work and the date of an invoice, which prompts caution in dating. Other contentual aspects, such as the notations used, can help finding an order for the manuscripts and files, but again without a high degree of precision. Besides, the establishment of the archive, as described above, adds to the difficulty: while it is certainly possible to date with a relative precision some of the folios, it cannot be applied to those surrounding them. 
Despite these difficulties and the lack of context and commentary from Dedekind's hand, the archive is extremely rich and offers great insights through these material traces of the research process. Dedekind's drafts are also very clean and easy to read, as they are well preserved, few things are scratched out and his handwriting is very readable.

\section{$2.2 \quad$ Dualgruppen in the published texts}

A Dualgruppe is defined by Dedekind in [Dedekind, 1897] in the following manner:

A system $\mathfrak{A}$ of things $\alpha, \beta, \gamma, \ldots$ is called a Dualgruppe, if there are two operations \pm , such that they create from two things $\alpha, \beta$, two things $\alpha \pm \beta$, that are also in $\mathfrak{A}$ and that satisfy [commutativity for + and -, associativity for + and - , and $\alpha \pm(\alpha \pm \beta)=\alpha$ (absorption)]. [Dedekind, 1897, 113, transl. in [Schlimm, 2011]].

A fact of great importance for Dedekind is that there exist examples of Dualgruppen in many domains of mathematics: Schröder's logic, modules, ideals, infinite Abelian groups, Galois groups, fields, number spaces [Dedekind, 1897, 113-114]. The broad range of application of the concept is, for Dedekind, a testimony of its usefulness and fruitfulness.

Dedekind defines a divisibility relationship, denoted by $\mathfrak{a}<\mathfrak{b}$ or $\mathfrak{b}>\mathfrak{a}$ (a a divisor of $\mathfrak{b}$ ) and defined by $\mathfrak{a}+\mathfrak{b}=\mathfrak{a}, \mathfrak{a}-\mathfrak{b}=\mathfrak{b}$. It satisfies the laws of a partial order relation. The fundamental properties (Grundgesetze) of modules display the "peculiar dualism" noticed by Dedekind when he introduced the operations.

Some Dualgruppen satisfy what Dedekind calls the Modulgesetz (modular law): for three modules $\mathfrak{d}, \mathfrak{m}, \mathfrak{p}$, if $\mathfrak{d}<\mathfrak{m}$, then

$$
(\mathfrak{m}+\mathfrak{p})-\mathfrak{d}=\mathfrak{m}+(\mathfrak{p}-\mathfrak{d}) .
$$

The Modulgesetz cannot be derived from the fundamental laws. It should be considered as extending them. Its name comes from the fact that Dualgruppen formed by modules (e.g. the Dualgruppe generated by three modules) satisfy this law. Dualgruppen satisfying this law are called "of the module type" (Dualgruppen vom Modultypus). An analog law is given for Dualgruppen generated by ideals and called Idealgesetz:

$$
(\mathfrak{b}+\mathfrak{c})-(\mathfrak{c}+\mathfrak{a})-(\mathfrak{a}+\mathfrak{b})=(\mathfrak{b}-\mathfrak{c})+(\mathfrak{c}-\mathfrak{a})+(\mathfrak{a}-\mathfrak{b}) .
$$

Finally, let me mention two notions which were initially introduced in module theory, and are used in the theory of Dualgruppen: the number of congruence classes in a module $\mathfrak{m}$ according to a module $\mathfrak{n}$ and denoted by $(\mathfrak{m}, \mathfrak{n})$, and the notion of chain (Kette) of modules. A chain of modules is a finite ordered (with $<$ ) sequence of at least two modules. ${ }^{12}$ I will

\footnotetext{
${ }^{12}$ This notion of Kette, which is also used in [Dedekind, 1894, 90], and was largely used in algebra after that, is not related to the concept introduced in [Dedekind, 1888] under the same name.
} 
concentrate, in this paper, on the operations,+- and the divisibility relation $<$, as they constitute the core of Dedekind's research.

An early version of the notion is alluded to in [Dedekind, 1894, 66], however Dedekind's researches on the matter started, as he recalls it himself, in the 1870s while he was investigating modules - which led him to notice the "dualism" between the GCD and LCM operations in [Dedekind, 1877b]. Twenty years passed between Dedekind's first and fundamental insight and the publication of articles on the subject. In the introduction of [Dedekind, 1897], he states that he was "busy with these questions" for several years and arrived at the notion of Dualgruppe through "efforts to obtain this theory from the smallest number of fundamental laws" [Dedekind, 1897, 113]. Dedekind's perfectionism and great attention to details are well known, and it is not surprising that he kept this work under wraps for such a long time. His Nachlass gives us insights into these twenty years.

\section{Exploring module theory through computations}

Computations seem to constitute a large part of the first moments of the research process, and keep appearing consistently throughout the twenty years of Dedekind's research. They appear to be explorations of the properties of modules and their operations through computation. By this exploration, Dedekind is experimenting, so to speak, and observing the laws of module theory. It is the introduction of a new notation that allowed Dedekind not only to go from mimicking arithmetic to actually computing with modules, but also to highlight the duality of the operations.

\subsection{Computations on modules}

Dedekind's exploration can take the form of small computations on numerical examples or particular cases. Some of them can be identified as being part of a larger investigation. Other stand on their own.

An example of such computations is the following, in which Dedekind works with finitely generated modules with a basis composed of two numbers. These modules, denoted by $[a, b]$ with $a$ and $b$ the elements forming the basis, are all the numbers of the form $a x+b y$. On the pages 3 to 8 of Cod. Ms. Dedekind XI 11-1, which can be dated from the 1890s, he starts with specific modules:

$$
\mathfrak{a}=[10,1+15 \omega], \mathfrak{b}=[15,1+10 \omega], \mathfrak{c}=[25,1+6 \omega] .
$$

Dedekind computes all the possible combinations of the three initial elements with + and

-. While doing so, he carefully points out the (divisibility) relationships between elements, such as $\mathfrak{c}-\mathfrak{a}=50(\mathfrak{a}+\mathfrak{c})$. He then does the same with more general such modules:

$$
\mathfrak{a}=\left[h a_{1}, 1+b c \omega\right], \mathfrak{b}=\left[h b_{1}, 1+a c \omega\right], \mathfrak{c}=\left[h c_{1}, 1+a b \omega\right]
$$


and again with $\mathfrak{a}=\left[h a_{1}, a_{2}+b c \omega\right]$, etc.

While not extremely complicated, the computations (which resemble an identification of indeterminate coefficients) are lengthy and require a lot of ingenuity and familiarity with the subject. Dedekind's Nachlass contains dozens of such pages, in which he calculates with finite modules, takes examples, particular cases, stops the calculation in what seems to be the middle, starts over later, and so on.

On many occasions, Dedekind focuses on more general investigations or studies of the divisibility relations and possible "permutations" between modules. There, Dedekind works with arbitrary modules (rather than finite modules or examples). A paradigmatic example, for this kind of research, are the operations between three modules $\mathfrak{a}, \mathfrak{b}, \mathfrak{c}$. The general computations appear to be mostly a reflection on the divisibility properties of modules, using the GCD and LCM operations, the order / divisibility relation, and classes of congruences. Drafts typically contain:

- Statement of the three arbitrary given modules and, in some cases, certain initial conditions (e.g. three modules $\mathfrak{a}, \mathfrak{b}, \mathfrak{c}$ with $\mathfrak{b}<\mathfrak{c}$ ); statement of the three GCDs and three LCMs for each two of these three modules.

- Computation of the GCD and LCM for three and more operands.

- Computations on the number of congruence classes and their relations to divisibility properties observed earlier or induced from these new computations; in the latter case, possible proofs of these new properties.

- Considerations on the order / divisibility relations for two or more operands, and statements on some chains of modules; in some cases, attempts at an exhaustive list of the chains in the case studied.

\subsection{Notations, duality and computations}

In his published works, Dedekind presented the "peculiar dualism" of the operations defined between modules as a key element for his researches. Duality comes forth as a motivation to study in more detail the operations between modules. As the research goes on, the mere interest in the duality of operations is replaced by the developments of module theory and later Dualgruppen. Yet, duality appears regularly as a sort of guiding light in the exploration of new aspects of module theory. More importantly, here, is the fact that insofar as Dedekind's research goes through a (long) phase of exploring the properties of modules and their operations through computations, the reflection on duality and the computations enrich each other. Hence, duality is a driving force for investigating the operations' properties, and at the same time, the computations allow to experiment on the said operations and to observe the duality's behavior. Duality is, thus, omnipresent in the computations themselves. 
Of particular interest to understand the mathematics in the making is the way Dedekind devised his notations and the layouts of his drafts, and elaborated tools to accompany and help him through the computations. One of the most notable features of Dedekind's modes of writing is the strong symmetry displayed in the notations and in the way computations are organized. Indeed, the notations are designed to emphasize, or maybe showcase this symmetry, this dualism: for three modules $\mathfrak{a}, \mathfrak{b}, \mathfrak{c}$, Dedekind often writes in a "dualistic" setting as he puts it:

$$
\begin{array}{c|c}
\mathfrak{b}+\mathfrak{c}=\mathfrak{a}^{\prime \prime \prime} & \mathfrak{b}-\mathfrak{c}=\mathfrak{a}_{3} \\
\mathfrak{a}+\mathfrak{c}=\mathfrak{b}^{\prime \prime \prime} & \mathfrak{a}-\mathfrak{c}=\mathfrak{b}_{3} \\
\mathfrak{a}+\mathfrak{b}=\mathfrak{c}^{\prime \prime \prime} & \mathfrak{a}-\mathfrak{b}=\mathfrak{c}_{3}
\end{array}
$$

And with

$$
\begin{gathered}
\mathfrak{a}+\mathfrak{b}+\mathfrak{c}=\mathfrak{d}^{\prime \prime \prime \prime} \text { and } \mathfrak{a}-\mathfrak{b}-\mathfrak{c}=\mathfrak{d}_{4} \\
\mathfrak{a}^{\prime \prime \prime}-\mathfrak{b}^{\prime \prime \prime}-\mathfrak{c}^{\prime \prime \prime}=\mathfrak{d}^{\prime} \text { and } \mathfrak{a}_{3}+\mathfrak{b}_{3}+\mathfrak{c}_{3}=\mathfrak{d}_{1}
\end{gathered}
$$

He then continues with, for example:

$$
\begin{gathered}
\mathfrak{a}^{\prime \prime}=\mathfrak{b}^{\prime \prime \prime}-\mathfrak{c}^{\prime \prime \prime}=\mathfrak{a}+\mathfrak{d}^{\prime} \text { and } \mathfrak{a}_{2}=\mathfrak{b}_{2}+\mathfrak{c}_{2}=\mathfrak{a}-\mathfrak{d}_{1}\left(\text { and so on for } \mathfrak{b}^{\prime \prime}, \mathfrak{c}^{\prime \prime} \text { and } \mathfrak{b}_{2}, \mathfrak{c}_{2}\right) \\
\mathfrak{a}^{\prime}=\mathfrak{a}+\mathfrak{a}_{3}=\mathfrak{a}+\mathfrak{d}_{1} \text { and } \mathfrak{a}_{1}=\mathfrak{a}-\mathfrak{a}^{\prime \prime \prime}=\mathfrak{a}-\mathfrak{d}^{\prime}\left(\text { and so on for } \mathfrak{b}^{\prime}, \mathfrak{c}^{\prime} \text { and } \mathfrak{b}_{1}, \mathfrak{c}_{1}\right)
\end{gathered}
$$

This example is taken from Cod. Ms. Dedekind X 11-2, p. 15r (approximative date: mid1890s). Both the organisation in columns, and the play of symmetry between the ' and the indexes (and the operations) give Dedekind the possibility to systematically work with this symmetry, and to put forward what he sometimes calls "dual couples". Indeed, while the notation changes slightly over time (for example, Dedekind sometimes starts with $\mathfrak{b}+\mathfrak{c}=\mathfrak{a}^{\prime}$ and $\mathfrak{b}-\mathfrak{c}=\mathfrak{a}_{1}$, then $\mathfrak{a}^{\prime \prime}=\mathfrak{a}^{\prime}-\mathfrak{b}^{\prime}$, etc.), the symmetry between the 'for the addition and the indexes for the subtraction is constant.

Results stated using this notation also display the "dualism" of module theory, such as, in Cod. Ms. Dedekind X 11-2, p. 15v:

$$
\begin{aligned}
& \text { I. }(\mathfrak{a}, \mathfrak{b})=(\mathfrak{a}+\mathfrak{b}, \mathfrak{b}) \\
& \text { II. }(\mathfrak{a}, \mathfrak{b})=(\mathfrak{a}, \mathfrak{a}-\mathfrak{b}) \\
& \text { (...) } \\
& \text { III. }(\mathfrak{b}, \mathfrak{c})=(\mathfrak{b}+\mathfrak{c}, \mathfrak{c})=\left(\mathfrak{a}^{\prime \prime \prime}, \mathfrak{c}\right) \\
& \text { (...) } \\
& \operatorname{IV} . \quad\left\{\begin{array}{l}
(\mathfrak{b}, \mathfrak{c})=\left(\mathfrak{b}, \mathfrak{b}^{\prime \prime \prime}\right)\left(\mathfrak{c}^{\prime \prime}, \mathfrak{c}\right) ;(\mathfrak{c}, \mathfrak{a})=\left(\mathfrak{c}, \mathfrak{c}^{\prime \prime \prime}\right)\left(\mathfrak{a}^{\prime \prime}, \mathfrak{a}\right) ;(\mathfrak{a}, \mathfrak{b})=\left(\mathfrak{a}, \mathfrak{a}^{\prime \prime \prime}\right)\left(\mathfrak{b}^{\prime \prime}, \mathfrak{b}\right) \\
(\mathfrak{c}, \mathfrak{b})=\left(\mathfrak{c}, \mathfrak{c}^{\prime \prime \prime}\right)\left(\mathfrak{b}^{\prime \prime}, \mathfrak{b}\right) ;(\mathfrak{a}, \mathfrak{c})=\left(\mathfrak{a}, \mathfrak{a}^{\prime \prime \prime}\right)\left(\mathfrak{c}^{\prime \prime}, \mathfrak{c}\right) ;(\mathfrak{b}, \mathfrak{a})=\left(\mathfrak{b}, \mathfrak{b}^{\prime \prime \prime}\right)\left(\mathfrak{a}^{\prime \prime}, \mathfrak{a}\right)
\end{array}\right.
\end{aligned}
$$

This gives :

$$
\text { V. }(\mathfrak{b}, \mathfrak{c})(\mathfrak{c}, \mathfrak{a})(\mathfrak{a}, \mathfrak{b})=\left(\mathfrak{a}, \mathfrak{a}^{\prime \prime \prime}\right)\left(\mathfrak{b}, \mathfrak{b}^{\prime \prime \prime}\right)\left(\mathfrak{c}, \mathfrak{c}^{\prime \prime \prime}\right)\left(\mathfrak{a}^{\prime \prime}, \mathfrak{a}\right)\left(\mathfrak{b}^{\prime \prime}, \mathfrak{b}\right)\left(\mathfrak{c}^{\prime \prime}, \mathfrak{c}\right)
$$


Since $(\mathfrak{b}, \mathfrak{c})=(\mathfrak{b}, \mathfrak{b}-\mathfrak{c})=\left(\mathfrak{b}, \mathfrak{a}_{3}\right)$, it follows "dualistically": 13

$$
\text { VI. }\left\{\begin{aligned}
&(\mathfrak{b}, \mathfrak{c})=\left(\mathfrak{b}, \mathfrak{b}_{2}\right)\left(\mathfrak{c}_{3}, \mathfrak{c}\right) ;(\mathfrak{c}, \mathfrak{a})=\left(\mathfrak{c}, \mathfrak{c}_{2}\right)\left(\mathfrak{a}_{3}, \mathfrak{a}\right) ;(\mathfrak{a}, \mathfrak{b})=\left(\mathfrak{a}, \mathfrak{a}_{2}\right)\left(\mathfrak{b}_{3}, \mathfrak{b}\right) \\
&(\mathfrak{c}, \mathfrak{b})=\left(\mathfrak{c}, \mathfrak{c}_{2}\right)\left(\mathfrak{b}_{3}, \mathfrak{b}\right) ;(\mathfrak{a}, \mathfrak{c})=\left(\mathfrak{a}, \mathfrak{a}_{2}\right)\left(\mathfrak{c}_{3}, \mathfrak{c}\right) ;(\mathfrak{b}, \mathfrak{a})=\left(\mathfrak{b}, \mathfrak{b}_{2}\right)\left(\mathfrak{a}_{3}, \mathfrak{a}\right)
\end{aligned}\right.
$$

And thus:

$$
\text { VII. }(\mathfrak{b}, \mathfrak{c})(\mathfrak{c}, \mathfrak{a})(\mathfrak{a}, \mathfrak{b})=\left(\mathfrak{a}, \mathfrak{a}_{2}\right)\left(\mathfrak{b}, \mathfrak{b}_{2}\right)\left(\mathfrak{c}, \mathfrak{c}_{2}\right)\left(\mathfrak{a}_{3}, \mathfrak{a}\right)\left(\mathfrak{b}_{3}, \mathfrak{b}\right)\left(\mathfrak{c}_{3}, \mathfrak{c}\right) .
$$

For Dedekind, V. and VII. present duality properties, but note that the equalities must be looked at diagonally: the first three (resp. last three) factors of the right-hand side of $\mathrm{V}$. correspond to the last three (resp. first three) factors of the right-hand side of VII.

The results in IV. to VII. come from I., II., III., (valid for the three modules $\mathfrak{a}, \mathfrak{b}, \mathfrak{c}$ ) and rely on similar properties of the number of congruence classes, which can be easily deduced from the multiple / divisor relationships that constitute the definition of the elements.

In addition, Dedekind introduces a way to number the equations that also display the dualism:

$$
\begin{aligned}
(\mathfrak{c}+\mathfrak{a})-(\mathfrak{a}+\mathfrak{b}) & =\mathfrak{a}+(\mathfrak{b}-\mathfrak{c}) \\
(\mathfrak{c}-\mathfrak{a})+(\mathfrak{a}-\mathfrak{b}) & =\mathfrak{a}-(\mathfrak{b}+\mathfrak{c})
\end{aligned}
$$

in which he uses a numbering of the results of the form $(a)$ and $\left(a^{\prime}\right)$ to denote the duality relation between two results. This numbering is also used in the published papers.

The modes of writing devised by Dedekind thus clearly put forward the duality between the two operations + and -. By doing so, they facilitate the manipulation of the said operations, as the duality can also be used to shorten some of the computations or proofs.

\subsection{Layouts and devices}

A second essential aspect in Dedekind's modes of writing are the layouts of his drafts. The presentation of the operations in two columns given p. 10 is often used by Dedekind, be it in organizing the results of computations or when drawing up lists of all the possible additions and subtractions for three modules. Such lists are part of a larger toolbox developed by Dedekind to accompany his computations.

One of the most common of these devices are tables: lists of all possible combinations, tables of divisors, of GCD / LCM, of divisibility, several of which are 28 lines and 28 columns. ${ }^{14}$ In such tables, this notation generally allows to really emphasize this dualism, as they display a perfect symmetry between GCDs and LCMs (or between divisor and multiples).

\footnotetext{
${ }^{13}$ Recall that $\mathfrak{a}^{\prime \prime}=\mathfrak{a}+\mathfrak{d}^{\prime}$ and $\mathfrak{a}_{2}=\mathfrak{a}-\mathfrak{d}_{1}$ (with $\mathfrak{d}^{\prime}=\mathfrak{a}^{\prime \prime \prime}-\mathfrak{b}^{\prime \prime \prime}-\mathfrak{c}^{\prime \prime \prime}$ and $\mathfrak{d}_{1}=\mathfrak{a}_{3}+\mathfrak{b}_{3}+\mathfrak{c}_{3}$ being dual of each other), thus being dual of each other (and so on for $\mathfrak{b}^{\prime \prime}, \mathfrak{c}^{\prime \prime}$ and $\mathfrak{b}_{2}, \mathfrak{c}_{2}$ ). The equalities are equivalent to $(\mathfrak{b}, \mathfrak{c})=(\mathfrak{b}, \mathfrak{a}+\mathfrak{c})\left(\mathfrak{c}+\mathfrak{d}^{\prime}, \mathfrak{c}\right)=\left(\mathfrak{b}, \mathfrak{b}-\mathfrak{d}_{1}\right)(\mathfrak{b}-\mathfrak{a}, \mathfrak{c})$

${ }^{14}$ For the Dualgruppe generated by 3 modules, see [Dedekind, 1900, 246-247].
} 
There are about 15 such tables standing on their own (often 28 lines by 28 columns), and many more (smaller ones) integrated into the computations or researches. Interestingly Dedekind does not draw a lot of different tables, but actually draws several times the same table with different notations, different organisations. These tables, which suggest that even more computations happened in non-preserved drafts, appear to be both an intermediary result, and a tool for the research.

The tables were an intermediary result insofar as they are a result of Dedekind's many computations, which are subsequently ordered and put in the said table. The fact that he made so many different versions suggests that he was looking for the best version, an optimized table to display the results. One of the most striking examples of this is a table given in Cod. Ms. Dedekind X 11-1, p. 18, which bears the traces of its own elaboration. Indeed, Dedekind first wrote the elements themselves, then replaced them by the notation he designed, for example:

\begin{tabular}{c|c|c|c|c|c}
$a^{\prime}$ & $b^{\prime}$ & $c^{\prime}$ & $d$ & $d$ & $d$ \\
\hline$a_{1}$ & $b_{1}$ & $c_{1}$ & $b_{1}+c_{1} a_{3}$ & $\epsilon_{1}+a_{1} b_{3}$ & $a_{1}+b_{1} c_{3}$ \\
\hline$a-a_{1} a^{\prime \prime}$ & $b-b_{1} b^{\prime \prime}$ & $e-c_{1} c^{\prime \prime}$ & $m^{\prime}$ & $m^{\prime}$ & $m^{\prime}$
\end{tabular}

It is thus possible, here, to observe the temporality of the elaboration of this table. Moreover, the complete table suggests that Dedekind was looking for a pattern, looking maybe for the clean symmetry which can be seen in the finished tables (see the table of GCD and LCM for three modules, published in [Dedekind, 1900, 246-247]).

As such, the tables appear to also be a device, for Dedekind, in conducting computations. They help understanding the computations, the patterns, the duality that appears in the computations and which can be difficult to visualize or understand without a graphical device such as this one. But the tables can also serve as a tool to verify the computations and to shorten them. At several occasions, Dedekind refers to the tables to avoid doing the same computations over and over again, and would say for example "according to the table of three modules, $c^{\prime \prime \prime}=a+b^{\prime \prime}$.

A second device elaborated by Dedekind to support his computations are diagrammatic representations of the notion of chain of modules. These diagrams present a striking resemblance with Hasse diagrams, the modern representation of lattices (see Figure 1). In the diagram reproduced below (which can be dated from the early 1890s), starting at the center, each link corresponds to the relation "being a divisor of": $\mathfrak{d}=\mathfrak{a}^{\prime}+\mathfrak{b}^{\prime}+\mathfrak{c}^{\prime}$, hence $\mathfrak{d}$ is a divisor of $\mathfrak{a}$, of $\mathfrak{b}$ and of $\mathfrak{c}$. Dedekind would note, in symbols, $\mathfrak{d}<\mathfrak{a}^{\prime}, \mathfrak{b}^{\prime}, \mathfrak{c}^{\prime}$.

Following the successive links from the center to the upper left, we obtain a chain of modules: $:^{15}$

$$
\mathfrak{d}<\mathfrak{a}^{\prime}<\mathfrak{b}^{\prime \prime \prime}<\mathfrak{b}_{2}<\mathfrak{b}<\mathfrak{b}^{\prime \prime}<\mathfrak{b}_{3}<\mathfrak{a}_{1}
$$

\footnotetext{
${ }^{15}$ It is likely that the $\mathfrak{a}_{3}$ indicated on the diagram is a mistake - as supported by the chain given a little further down in the manuscript.
} 


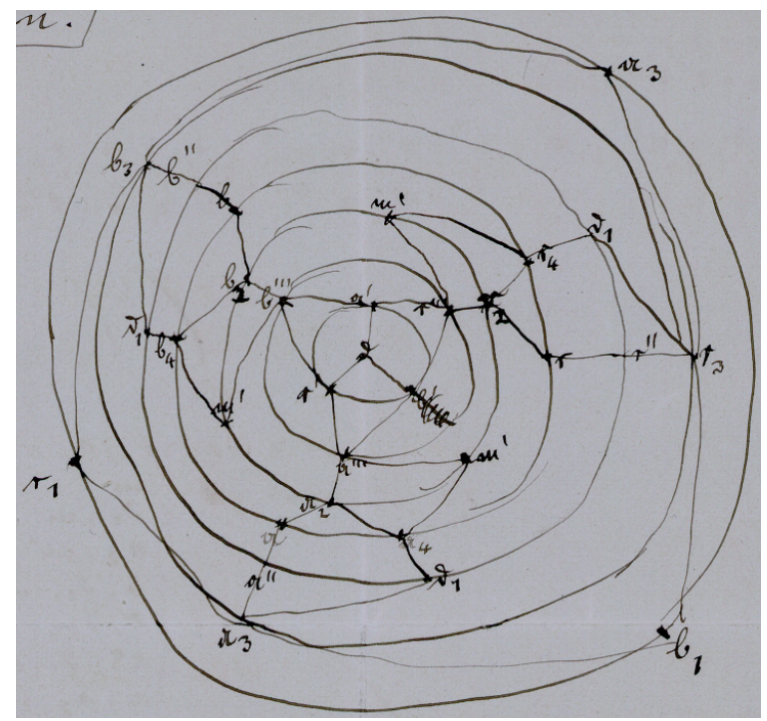

Figure 1: Cod. Ms. Dedekind XI 11-1 24

with

$$
\begin{gathered}
\mathfrak{d}=\mathfrak{a}^{\prime}+\mathfrak{b}^{\prime}+\mathfrak{c}^{\prime} \\
\mathfrak{a}^{\prime}=\mathfrak{b}^{\prime \prime \prime}+\mathfrak{c}^{\prime \prime \prime} \\
\mathfrak{b}^{\prime \prime \prime}=\mathfrak{b}_{2}+\mathfrak{c}_{2} \\
\mathfrak{b}_{2}=\mathfrak{b}+\mathfrak{b}_{1} \\
\mathfrak{b} \\
\mathfrak{b}^{\prime \prime}=\mathfrak{b}-\mathfrak{b}^{\prime} \\
\mathfrak{b}_{3}=\mathfrak{b}^{\prime \prime}-\mathfrak{c}_{2}=\mathfrak{b}^{\prime \prime}-\mathfrak{a}_{2}=\mathfrak{a}_{1}+\mathfrak{c}_{1} \\
\mathfrak{a}_{1}=\mathfrak{b}_{3}-\mathfrak{c}_{3}
\end{gathered}
$$

The notation for the addition and subtraction, with the symmetry between ' and indexes, is also clearly observable in the chain. For a perfect symmetry, one would need to add $\mathfrak{m}=\mathfrak{a}_{1}-\mathfrak{b}_{1}-\mathfrak{c}_{1}$, but this does not appear in Dedekind's diagram. This may be because of a lack of space, or of a good way to draw all the links, since $\mathfrak{m}$ does appear in the list of chains that go with the diagram. In any case, it seems clear that the notation was designed for computations, and not for the diagrams.

While chains of modules are defined and studied in the published papers, Dedekind never reproduced the diagrams. In the drafts, though, they do appear at several occasions. This is not a systematic feature of Dedekind's research on modules, but it is nevertheless very striking. Indeed Dedekind spoke openly against using visual representations in mathematics, if it were not for purely pedagogical or heuristic reasons. He underlined, in [Dedekind and Weber, 1882], that when defining the notion of a point of a Riemann 
surface, one should not appeal to a geometric representation because it "does not make comprehension much easier" [Dedekind and Weber, 1882, 94]. He also wrote, in the preface of [Dedekind, 1872], that it was acceptable to use the straight line as an analogy for the linear continuum, as long as it wasn't anything more than an helpful analogy, a heuristic aid. Using visualisation or geometric representation can be acceptable to better understand a subject or introduce it in teaching, but it should not be the basis of a definition or a demonstration.

It seems clear that diagrams appeared, in Dedekind's drafts, as a (temporary) aid to try to understand the properties of the order / divisibility relation for modules. When Dedekind sets himself the task of listing or studying all the possible chains generated by the operations between three modules, the amount and length of the inequalities becomes quickly overwhelming. At some points, in the drafts, Dedekind drew little arrows between the elements of some chains, in what could be an attempt to better visualize the relationships. ${ }^{16}$ It seems that the diagrams are the next step to indeed visualize the order relationship(s) between modules, the chains and their length, as well as a certain structure build by the order. However, Dedekind struggles to find the best way to actually represent all the links in a readable way, and in particular in way that would showcase the symmetry between the elements.

Dedekind was thus exploring the properties of modules and their operations, using computations as a way to experiment, to better understand the divisibility laws of modules by manipulating simple cases, testing on progressively more general cases. A slow process of generalization arises through these repeated computations: numerical examples, resolution of problems, particular cases with arbitrary elements, more general particular cases, attempts at a completely general approach... This process gives Dedekind key elements to observe the properties and laws verified by the operations between modules, and identify which properties are generally valid, and which laws are fundamental, i.e. cannot be deduced from other laws (e.g. associativity). In this process, the duality displayed by the operations, and put forward by the notations and layouts elaborated by Dedekind, is a tool for the conceptual clarification of (what will become) Dualgruppen. Noticed by Dedekind as a "peculiar" property of the operations, interesting enough to justify further investigating their properties, duality acts as a guiding light to explore new aspects of module theory. Indeed, as the next paragraph shows, duality is at the core of Dedekind's interest for the laws and properties of the operations between modules and their generalization.

\footnotetext{
${ }^{16}$ I am grateful to Karine Chemla for pointing this out to me.
} 


\section{On the operations, their (slowly gained) generality and their duality}

\subsection{Generalization of the operations}

In his search for the fundamental properties, Dedekind slowly engages in a generalization process, in which he tries to detach the operations from their interpretation in module theory. While his position on the matter is not completely clear, as he often goes back to module theory, there are distinct attempts in the direction of more general operations. In particular, Dedekind considers the operations as applying to other objects than modules, most often groups or ideals. The computations are thus conducted in different frameworks (albeit not too different, as Dedekind thought of modules as types of groups, and ideals as types of modules). The computations and Dedekind's overall strategy remain the same in substance. Some new results appear, such as what Dedekind later calls the Idealgesetz (see p. 8).

In the meantime, Dedekind also carefully read Schröder's Vorlesungen über die Algebra der Logik, in which Schröder aims at developing a logical calculus. ${ }^{17}$ Schröder's "calculus of classes" relies on laws which are similar to the axioms for a bounded lattice, and so to Dedekind's Dualgruppen. Dedekind's notes on Schröder's book show his enthusiasm for the work, and notably for recognizing the same duality as in his own work. Later, he cites Schröder's theory when investigating a "logical", "more general" approach (and as an example for the general concept of Dualgruppe in [Dedekind, 1897, Dedekind, 1900]). There is a strong possibility that the reading of Schröder's work could have had a significant impact on Dedekind's reflection on Dualgruppen.

Another symptom of Dedekind's wish to generalize his research are his hesitations between different notations for the operations: + and,$- \varphi$ and $\psi$, and + and $\vee$. The $\vee$ (which replaces the -) is only found in a couple of manuscripts, which can be dated from the late 1870s. For the two other notations, the choice seems to be context-related. Indeed, the + and the - are related to the arithmetical study of module theory, introduced in number theoretical works, and follow Dedekind's initial desire to mimic arithmetic in module (and ideal) theory. The $\varphi$ and $\psi$ are explicitly introduced as a step in the development of a "more general theory". These notations are usually used by Dedekind for 'set' theory, which he calls "logic", as in [Dedekind, 1888] ${ }^{18}$ to which he explicitly refers in his drafts. The approach using these notations abstracts the individual nature of the elements. In Cod. Ms. Dedekind X-11-2, p. 54 (ca. mid-1890s), Dedekind starts with computations for

\footnotetext{
${ }^{17}$ See [Peckhaus, 1994].

${ }^{18}$ Note that other uses of "logic" are made with reference to Schröder.
} 
three modules and then states:

$$
\left.\begin{array}{rllllllllll}
\text { One replaces }+, & -, & a, & b, & c, & b^{\prime \prime \prime}, & c^{\prime \prime \prime}, & b_{3}, & c_{3}, & d^{\prime}=d_{1} \\
\text { by } \quad \phi, & \psi, & a, & m, & d, & d^{\prime \prime \prime}, & d^{\prime}, & m^{\prime}, & m^{\prime \prime \prime}, & \underline{d^{\prime \prime}=m^{\prime \prime}}
\end{array}\right\} \begin{aligned}
& \text { NB! Consequence of the } \\
& \text { special module theory }
\end{aligned}
$$

The "Nota Bene" refers only to the underlined property. The title of the following investigations is "More general (logical) theory", and presents a theory whose elements are of unspecified nature. These notations are used in several drafts, first in researches on a "(more) general theory" or a "generalization of a part of module theory" in which he conducts the same computations as before, but reformulated with the new notations and without any reference to the individual nature of either the elements on which he computes, nor the operations themselves. The $\varphi$ and $\psi$ are also used later in researches on a general concept of Dualgruppe. In the published papers, however, Dedekind uses + and - .

Step by step, then, Dedekind increases the generality of the operations. He attempts to study them not as GCD and LCM of modules strictly speaking, but as indeterminate binary operations, a way to combine elements whose individual nature is also left indeterminate, and which satisfy given fundamental laws. Here, the researches are taken away from modules themselves, and the interest is moving towards the operations considered independently from the nature of the operands. Once the operations are not defined by the modules, the duality property stops being a property of modules, to become a property of the operations, independently from any interpretation.

\section{2 "On the dualism in module theory"}

Some of Dedekind's manuscripts study more explicitly the dualism of the operations, and it appears clear that he is questioning the systematicity and the origins of this duality. In the pages 13-14 of Cod. Ms. Dedekind X 11-1, which can be dated from the early to mid-1890s, Dedekind starts by studying the divisibility laws for three modules $\mathfrak{m}, \mathfrak{d}, \mathfrak{p}$, such that $\mathfrak{d}$ divides $\mathfrak{m}$. The two first pages are covered with computations for the divisibility of the three modules (notably all possible additions and subtractions), tables of GCDs, LCMs, multiples, and the systematic verification of properties of divisibility. These considerations end with a short paragraph, which seems to be a conclusion of the previous computations, and is titled "The source of the dualism" :

The source of dualism in the theory of modules, that is, the following theorem: I. If $\mathfrak{m}$ is divisible by $\mathfrak{d}$, then $(\mathfrak{m}+\mathfrak{p})-\mathfrak{d}=\mathfrak{m}+(\mathfrak{p}-\mathfrak{d})$ amounts to what follows (for the sign + ):

II. If $\mathfrak{m}+\mathfrak{d}=\mathfrak{d}, \mathfrak{a}+\mathfrak{m}+\mathfrak{p}=\mathfrak{m}+\mathfrak{p}, \mathfrak{a}+\mathfrak{d}=\mathfrak{d}$ then there exists at least one element (module) $\mathfrak{q}$ verifying the conditions

$$
\mathfrak{q}+\mathfrak{p}=\mathfrak{p}, \mathfrak{q}+\mathfrak{d}=\mathfrak{d}, \mathfrak{a}+\mathfrak{m}+\mathfrak{q}=\mathfrak{m}+\mathfrak{q}
$$


Or again (if one sets $\mathfrak{d}=\mathfrak{m}+\mathfrak{p}^{\prime}$ )

III. If $\mathfrak{a}+\mathfrak{m}+\mathfrak{p}=\mathfrak{m}+\mathfrak{p}, \mathfrak{a}+\mathfrak{m}+\mathfrak{p}^{\prime}=\mathfrak{m}+\mathfrak{p}^{\prime}$, then there is at least one element $\mathfrak{q}$ verifying the conditions:

$$
\mathfrak{q}+\mathfrak{p}=\mathfrak{p}, \mathfrak{q}+\mathfrak{m}+\mathfrak{p}^{\prime}=\mathfrak{m}+\mathfrak{p}^{\prime}, \mathfrak{a}+\mathfrak{m}+\mathfrak{q}=\mathfrak{m}+\mathfrak{q}
$$

But this theorem (I or II or III) is not in any way a necessary consequence of the three laws of pure addition:

IV. $\mathfrak{a}+\mathfrak{a}=\mathfrak{a}, \mathfrak{a}+\mathfrak{b}=\mathfrak{b}+\mathfrak{a},(\mathfrak{a}+\mathfrak{b})+\mathfrak{c}=\mathfrak{a}+(\mathfrak{b}+\mathfrak{c})$.

The identity stated here as the "source" of duality is the Modulgesetz of the published papers. ${ }^{19}$ This law is at the center of many of the drafts around the same period. For many pages, Dedekind explores and manipulates the operations in different ways, from mere computations (whose beginning and end can be difficult to locate) to attempted proofs, sometimes deemed insufficient. It seems that, at this point, Dedekind hasn't made the step of trying to apply the operations to other elements than modules. Once he does so, it becomes clear that the law presented as the "source of dualism" depends on properties of modules, which leads him to rename it, and attribute a different status to it. The Modulgesetz becomes an additional law that can be added to the initial conditions for studying the operations (and, later, the Dualgruppe).

More developed reflections on duality in module theory are found in some of the earliest fully written drafts, which also appear to be Dedekind's first attempts at a general written presentation of his work. The manuscript Cod. Ms. Dedekind XI 1, 1-27 was initially entitled On the dualism in module theory. Dedekind later corrected it to be On Dualgruppen. It must be read, Dedekind indicates, with a shorter manuscript entitled Some propositions on Modul-Gruppen (Cod. Ms. Dedekind XI 1, 29-32). These texts can be dated from between 1894 and 1897. In On the dualism in module theory, Dedekind uses the $\varphi$ and $\psi$ notations. In Some propositions on Modul-Gruppen, he uses the + and the - . I will consider the second one in the next section.

Despite its initial title, On the dualism in module theory seems to try to detach the investigation from the specific nature of modules and adopt a more general approach:

I have often noticed a curious dualism, which appears in the theory of modules. $^{*}$ The same is repeated so frequently in other domains of research, that it seems beneficial to understand the general laws of combinations [VerknüpfungsGesetze] which prevail in this theory independently of the initial premises on which it is grounded. If such is the case, and that we replace the signs + and -

\footnotetext{
${ }^{19}$ Dedekind's statement about the non-existence of a consequence link between the laws of addition and the Modulgesetz is in his published papers (see p. 7). A notable difference, in the published papers, is that the absorption law replaces the idempotence property, which is derived from the absorption law.

*Dirichlet's Vorlesungen über Zahlentheorie, fourth edition, §169.
} 
used to designate the greatest common divisor and the least common multiple of two modules, by $\varphi, \psi$, then only the following hypotheses remain:

In a finite or infinite system $S$ of elements $a, b, c, \ldots$ whose meaning is left completely indeterminate, there are two kinds of combinations $\varphi$ and $\psi$, which from any two identical or distinct elements $a, b$ always produce two completely determined elements of the same system $S$ designated by $a \varphi b, a \psi b$. They obey the following six laws

$$
\begin{array}{cc}
a \varphi=a & (1) \\
a \varphi b=b \varphi a & (2)(1) \\
(a \varphi b) \varphi c=a \varphi(b \varphi c) & (3)(2) \\
a \varphi a=a & \left(1^{\prime}\right) \\
a \psi b=b \psi a & \left(2^{\prime}\right)\left(1^{\prime}\right) \\
(a \psi b) \psi c=a \psi(b \psi c) & \left(3^{\prime}\right)\left(2^{\prime}\right) \\
a \psi(a \varphi b)(a \varphi b) \psi a=a & (4)(3) \\
a \varphi(a \psi b)(a \psi b) \varphi=a & \left(4^{\prime}\right)\left(3^{\prime}\right) .
\end{array}
$$

(Cod. Ms. Dedekind XI 1, 1-2)

The striken out laws show us the exact moment when Dedekind realized - as he explains in a note on the back of the page - that the idempotency could be deduced from the absorption law and was, thus, not a fundamental property.

The notation and layout for the laws put forward, as I mentioned previously, their dualism. Duality appears, in this paper, as an essential property of the operations, and as such, it is considered a core property of the theory. Its status stays relatively unclear, though. The initial title suggests that Dedekind is going to study the dualism itself, or at least tell us how or maybe why it is significant. This is not the case. Dedekind does explain the dualism evoked in the title:

Two of these laws, designated by $(\alpha)$ and $\left(\alpha^{\prime}\right)$, correspond to each other dualistically in the sense that they can be changed one into the other by switching $\varphi$ and $\psi$. Consequently, the dual transformation of the conditions of any proposition of our research always results in a second proposition, which is the dual equivalent of the first one, which itself, reciprocally, results from the second one in the same way, or can also very well be identical to it. (Cod. Ms. Dedekind XI 1, 2)

He makes the same remark when defining the order relation. Throughout the paper, the duality of the theorems is underlined by stating systematically each law for both operations and numbering them $(\alpha)$ and $\left(\alpha^{\prime}\right)$. If the duality is emphasized, and sometimes used in proofs, it is not justified at any point.

The "groups" defined by Dedekind do not have a name yet, which suggests that the theory he is developing, that of Modulgruppen and later Dualgruppen, has still to reach maturity. Dedekind endeavors to keep a general (not related to modules) approach throughout 
his manuscript. He gives properties of the operations and of the order relation without references to the nature of the operations or operands. He often refers to modules as an example. However, it appears to be more than a simple example, since in the paragraphe in which he studies the modular law, i.e. $\mathfrak{m}$ divisible by $\mathfrak{d}$, then $(\mathfrak{m}+\mathfrak{p})-\mathfrak{d}=\mathfrak{m}+(\mathfrak{p}-\mathfrak{d})$, he states that it is "in fact necessary" to "go back to the concept of module" in order to prove that this law is independent from the fundamental defining laws (Cod. Ms. Dedekind XI 1, 10). While Dedekind has noticed that this property is not "the source of the dualism" (which is rather a property of the operations) but a property specific to modules (in modern terms, it is the defining property of modular lattices), he hasn't, yet, named the property the Modulgesetz, the modular law.

\section{Groups, groups of modules and Dualgruppen}

The definition given in the text above is about a system in which are defined internal operations or combinations. It is, of course, not the same as defining a system by the property that it is closed under internal operations, which is the case in the definition of a Dualgruppe. The passage from working on operations between modules (and often all the modules generated by applying these operations to a finite number of modules) to considering systems of modules closed under these operations first appears in the inbetween notion of Modulgruppe, group of modules.

\subsection{Groups of modules}

The methods used by Dedekind throughout his researches are strikingly reminiscent of methods used to study abstract groups or groups of permutations at the time: studying the internal structure, drawing up tables, lists of elements... This kind of strategy was relatively common in the late 1800s. Dedekind's drafts often suggest that he was mainly working in module theory and going towards building and studying a 'group' of modules. Still, the process towards the notion of "groups of modules" is (again) slow, stepwise and not linear.

Rather than stating a general definition of a Modulgruppe and studying the general theory of such groups, Dedekind gives himself a lot of exercises (Aufgaben) to solve the construction a given group. His researches take, here, two directions: the study of groups of modules, and the study of groups satisfying "modular laws".

When working on groups of modules, Dedekind studies the construction of determined groups, such as "Construction of a group of three initially given arbitrary modules $\mathfrak{a}, \mathfrak{b}, \mathfrak{c}$ " (sometimes adding divisibility conditions). Interestingly, in these Aufgaben, Dedekind conducts the same computations as in module theory: the calculation of all the possible combinations of + and - are the same, but it is presented as the calculation of all the elements of the groups. These elements are subsequently listed or organized in a table, divisibility / order properties are studied, etc. 
When working on groups satisfying the so-called modular laws, Dedekind considers what he calls a "modulartige System". For example, in Cod. Ms. Dedekind XI 2 p. 47-50, which can be dated from after 1895, he defines a "modular system" as a system satisfying the following laws:

$$
\begin{aligned}
& (c \varphi a) \psi(a \varphi b)=a \varphi(b \psi(c \varphi a)) \\
& (c \psi a) \varphi(a \psi b)=a \psi(b \varphi(c \psi a))
\end{aligned}
$$

These laws are the ones Dedekind first found in module theory in the late 1870s (see p. 2). Dedekind, here, wants to "build the group of 28 elements" satisfying these laws. Hence, he builds a group generated by three arbitrary elements that satisfy given laws which he knows to be characteristic of modules. Dedekind then computes all the elements, defines the order relation "which can be called divisibility for short", and so on.

The notion of Modulgruppe is formally defined for the first time in the second manuscript mentioned above, Some propositions on Modul-Gruppen:

A system $M$ of modules is called a group when the modules $a+b, a-b$ formed by any two modules $a, b$ belong to the same system. (Cod. Ms. Dedekind XI 1, 29)

The hyphen in the name and definition illustrate clearly the construction of the concept: Dedekind operated the important conceptual move of going from operations between modules to sets of modules closed under these operations - groups, in Dedekind's relatively wide idea of it. ${ }^{15}$ In the rest of the draft, Dedekind studies in detail the laws of divisibility for Modul-Gruppen, and introduces notions such as the number of congruence classes or chains of modules. At this point, the concept of a group of modules is still a concept pertaining to module theory, and not an instance of a more general concept as it will be after the introduction of Dualgruppen.

While the computations are similar over and over again, it is extremely significant that Dedekind went from studying three modules and their operations to building a group of modules formed or closed under these operations. With the definition of Modul-Gruppen, Dedekind thus undertook the crucial passage to what he calls the "higher level" of sets ([Dedekind, 1871, 399]). This action is essential here, and for many of Dedekind's works.

\subsection{The final steps towards Dualgruppen}

In On the dualism in module theory and Some propositions on Modul-Gruppen, Dedekind wants to merge (1) a very general theory based on the duality of operations defined between two sets (whose nature is indifferent). Even though the links to module theory are still strong, the definitions and properties are presented in a general manner, to underline the possibility of applications to other domains. And (2) an investigation into the theory of

\footnotetext{
${ }^{15}$ For Dedekind, any set of elements closed under a binary operation was considered a group.
} 
modules and groups formed by modules, which clearly builds on Dedekind's previous (not necessarily published) works on module theory and elaborates a new concept, Modulgruppen. Dedekind's Dualgruppe is the result of this fusion: on one hand, the generalization from modules to any elements satisfying the fundamental laws, and on the other hand, the passage to the level of sets.

There is a noticeable shift from the drafts mentioned above to the published version, which can already be seen in the change of title suggested by Dedekind. Dedekind went from studying the duality of operations in module theory to a concept of group whose core property is this duality - the Dualgruppe. Hence, the theory is not about a duality of operations anymore, but about a certain group whose operations present a duality.

The work on the Dualgruppe generated by three modules, which runs through Dedekind's manuscripts, also exhibits these changes. It started with computations on finitely generated modules, as exemplified earlier in this paper. Dedekind then wrote several drafts on "the theory of three modules", exploring the laws and properties through more general computations (for any three modules) and regularly proposing "better presentations". Finally, he studied the "Dualgruppe of $a, b, c$ " with various initial hypotheses: with and without Modulgesetz, with special divisibility restrictions, for ideals, or in general.

\section{Conclusion}

In this paper, I illustrated how Dedekind gradually built his Dualgruppe theory through many layers of computations by exploring the various possibilities offered by operations defined between modules, and by their duality. He used series of examples, tables, calculations, special cases, and gradual generalizations to delve into the subject, and devised tables and diagrams as graphical tools to help and accompany him in this exploration. The study of his manuscripts, which reveal concealed layers of mathematical practice that do not appear in published papers, show two main directions of this slow building of a new theory. On one hand, there is a progressive generalization, happening inside the computations themselves, moving the theory away from module theory, its place of birth, and towards a more general theory. On the other hand, one observes a shift from studying properties of operations to studying groups closed under these binary operations. In this move, which goes together with the generalization, the duality of the operations, which prompted Dedekind's research, eventually becomes a defining, characteristic property of Dualgruppen.

\section{References}

[Barberousse and Pinon, 2003] Barberousse, A. and Pinon, L., editors (2003). Genesis 20 «Écriture scientifique ». Presses de l'Université Paris-Sorbonne, Paris. 
[Birkhoff, 1934] Birkhoff, G. (1934). Note on the paper "On the combination of subalgebras". Mathematical Proceedings of the Cambridge Philosophical Society, 30(2):200.

[Chemla, 1995] Chemla, K. (1995). Histoire des sciences et matérialité des textes. Enquête, $1: 167-182$.

[Chemla, 2004] Chemla, K., editor (2004). History of Science, History of Text. Springer, Boston Studies in the Philosophy of Science 238, Dordrecht.

[Corry, 2004] Corry, L. (2004). Modern Algebra and the Rise of Mathematical Structures. Springer, New York.

[de Biasi, 2011] de Biasi, P.-M. (2011). Génétique des textes. CNRS éditions, Paris.

[Dedekind, 1858] Dedekind, R. (1856-1858). Eine Vorlesung über Algebra. In [?], pages 59-108.

[Dedekind, 1871] Dedekind, R. (1871). Über die Composition der binären quadratischen Formen, Xth Supplement. In Vorlesungen über Zahlentheorie, 2nd edition, pages 380497. Partially repr. in [Dedekind, 1932] III, pages 223-262.

[Dedekind, 1872] Dedekind, R. (1872). Stetigkeit und irrationale Zahlen. In [Dedekind, 1932], volume III, pages 315-335.

[Dedekind, 1877a] Dedekind, R. (1876-1877a). Théorie des nombres entiers algébriques. Published in five parts in Bulletin des Sciences Astronomiques et Mathématiques: BSAM 11 (1876), pages 278-288; BSAM Sér. 2(1):1 (1877), pages 17-41, 69-92, 144-164, and 207-248. Partially repr. in [Dedekind, 1932] III, pages 262-296. Engl. transl. in [?]. All references to [?].

[Dedekind, 1877b] Dedekind, R. (1877b). Über die Anzahl der Ideal-Klassen in den verschiedenen Ordnungen eines endlichen Körpers. In [Dedekind, 1932], volume I, pages 105-159.

[Dedekind, 1888] Dedekind, R. (1888). Was sind und was sollen die Zahlen? In [Dedekind, 1932] III, pages 335-392.

[Dedekind, 1894] Dedekind, R. (1894). Über die Theorie der algebraischen Zahlen, XIth Supplement. In Vorlesungen über Zahlentheorie, 4th edition, pages 434-657. Repr. in [Dedekind, 1932] III, pages 1-222.

[Dedekind, 1897] Dedekind, R. (1897). Über Zerlegungen von Zahlen durch ihre größten gemeinsamen Teiler. In [Dedekind, 1932], volume II, pages 103-147.

[Dedekind, 1900] Dedekind, R. (1900). Über die von drei Moduln erzeugte Dualgruppe. In [Dedekind, 1932], volume II, pages 236-271. 
[Dedekind, 1932] Dedekind, R. (1930-1932). Gesammelte mathematische Werke. 3 vols. Ed. by Robert Fricke, Emmy Noether and Öystein Ore. F. Vieweg and Sohn, Vieweg, Braunschweig. Re-ed. Chelsea Publishing Co. (1968), New York.

[Dedekind, 1870] Dedekind, R. (around 1870). Allgemeine Sätze über Räume. in [Dedekind, 1932] II, pages 353-356.

[Dedekind and Weber, 1882] Dedekind, R. and Weber, H. (1882). Theorie der algebraischen Funktionen einer Veränderlichen. In Journal für reine und angewandte Mathematik, Bd. 92, S. 181-290. Repr. in [Dedekind, 1932] I, pages 238-351.

[Dugac, 1976] Dugac, P. (1976). Richard Dedekind et les fondements des mathématiques. Vrin, Paris.

[Edwards et al., 1982] Edwards, H., Neumann, O., and Purkert, W. (1982). Dedekind's 'Bunte Bemerkungen' zu Kroneckers 'Grundzüge'. Archive for History of Exact Sciences, 27(1):49-85.

[Edwards, 2010] Edwards, H. M. (2010). The Algorithmic Side of Riemann's Mathematics. In Bott, R. and Kotiuga, P., editors, A Celebration of the Mathematical Legacy of Raoul Bott, CRM proceedings \& lecture notes, Providence. American Mathematical Society.

[Ehrhardt, 2012] Ehrhardt, C. (2012). Itinéraire d'un texte mathématique. Réélaborations d'un mémoire de Galois au XIXe siècle. Hermann, Paris.

[Grésillon, 2016] Grésillon, A. (2016). Éléments de critique génétique - Lire les manuscrits modernes. CNRS Collection : Textes \& manuscrits.

[Hersch, 1991] Hersch, R. (1991). Mathematics has a front and a back. Synthese, 8(2):127133.

[Holmes et al., 2003] Holmes, F. L., Renn, J., and Rheinberger, H.-J., editors (2003). Reworking the Bench. Research Notebooks in the History of Science. Number 7 in Archimedes New Studies in the History and Philosophy of Science and Technology. Kluwer Academic Publishers, New York, Boston, Dordrecht, London, Moscow.

[Mehrtens, 1979] Mehrtens, H. (1979). Die Entstehung der Verbandstheorie. Hildesheim, Gerstenberg.

[Peckhaus, 1994] Peckhaus, V. (1994). Wozu Algebra der Logik? Ernst Schröders Suche nach einer universalen Theorie der Verknüpfungen. Mod. Log., 4(4):357-381.

[Schlimm, 2011] Schlimm, D. (2011). On the creative role of axiomatics. The discovery of lattices by Schröder, Dedekind, Birkhoff, and others. Synthese, 183(1):47-68. 
[Wussing, 1984] Wussing, H. (1984). The genesis of the abstract group concept. A contribution to the history of the origin of abstract group theory. MIT Press, Cambridge, Mass., and London. 
Acknowledgements This research was supported by the DFG-Forschungsprojekt " $D u$ alität - ein Archetypus mathematischen Denkens". I am grateful to the members of the project for their useful remarks. I am also deeply indebted to the members of the "Histoire des sciences, histoire du texte" group of the Laboratoire SPHERE, for many insightful and inspiring discussions. I would finally like to thank two anonymous referees for their comments on this paper. 